\title{
An Education Program Using Tera-Byte NEA Observation Data
}

\author{
A. Asami, D.J. Asher, T. Hashimoto, S. Isobe, S. Nishiyama, \\ Y. Ohshima, J. Terazono, T. Urata, and M. Yoshikawa
}

Japan Spaceguard Association

\begin{abstract}
There are three wide-field telescopes at the Bisei Spaceguard Center operated by the Japan Spaceguard Association. These telescopes are dedicated to detect near-earth asteroids and produce several tera-byte data per month. Since these data contain many main-belt asteroids, we will use them for an education program that will allow school pupils and the general public to find new main-belt asteroids. We are now developing a new software for its purpose.
\end{abstract}

\section{Introduction}

There are several teams developing automatic remote operation telescopes called robotic telescopes. These telescopes make day-time observing possible because the telescopes are far apart in longitudes, well suited for day-time astronomy education. However, within this system, one telescope is occupied by only one team and many telescopes should be prepared to make many teams satisfy. This situation brings a limitation on usage of the telescope system.

An effective way in astronomical education is to offer pupils and others good astronomical exercises. If these exercises contain only ready-made data, their interest in astronomy can not continue to flourish. It is certainly better to use original data than ready-made data. Our telescopes are producing so many original data which can be distributed to more than a few thousand teams per month. Therefore, we are developing a new asteroid detection software by which even non-professionals can find a number of moving objects from several given images obtained by the BATTeRS (Bisei Asteroid Tracking Telescope for Rapid Survey) team under the Japan Spaceguard Association.

Here, we will describe our telescope system and the newly developed educational software.

\section{The Near-Earth Asteroid (NEA) Project at the Bisei Spaceguard Center}

During the last decade, a possibility of NEA collisions with the Earth and their hazardous consequences to human-beings has been studied and naturally we first have to detect these NEAs and determine their orbits in order to avoid the hazard. At this stage there are 5 US teams which work mainly for this detection observations. However, these are not enough to detect all the NEAs with a 


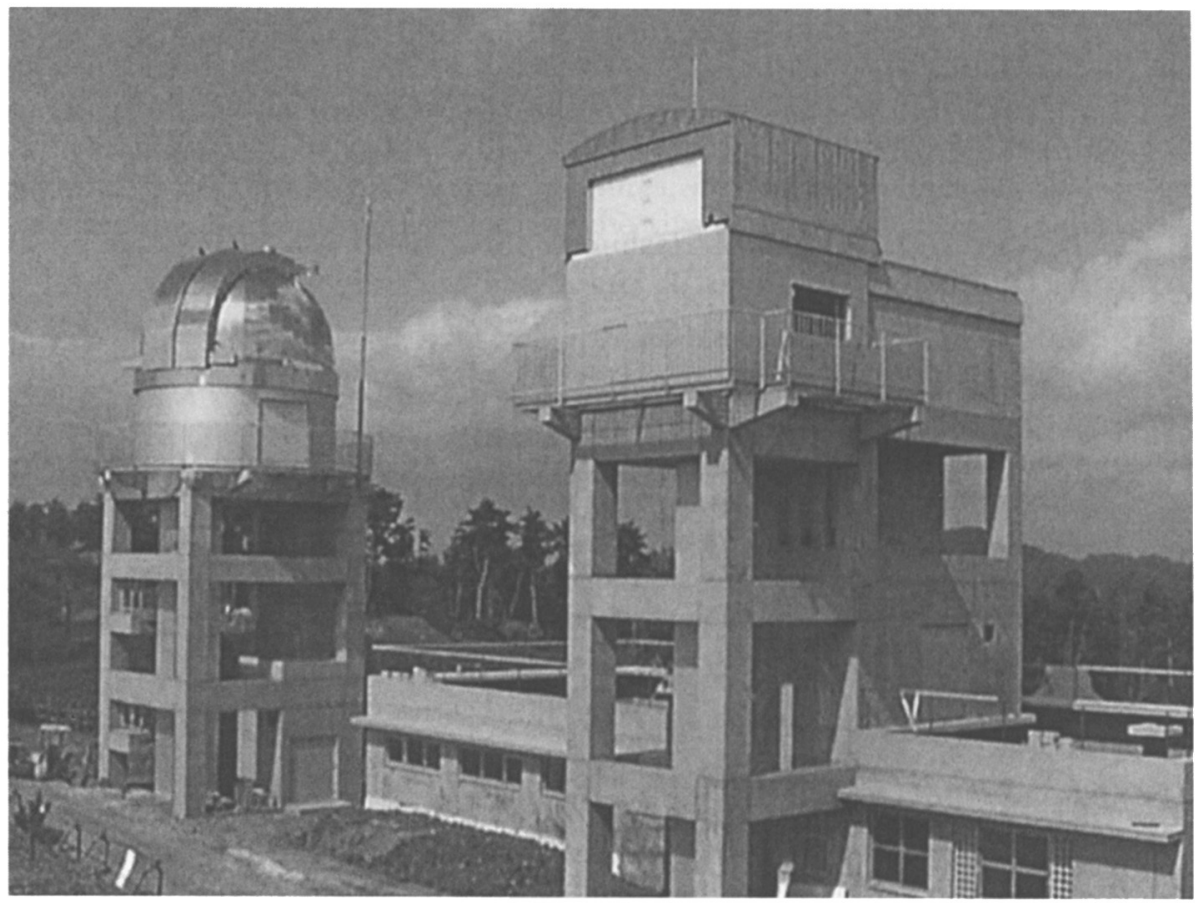

Figure 1. An over-view of the Bisei Spaceguard Center. A 1-m telescope will be set in the dome and the $25-\mathrm{cm}$ and $50-\mathrm{cm}$ telescopes are operated in the sliding roof building.

diameter large than $0.5 \mathrm{~km}$ within decades, and therefore it is highly desirable within the NEA community to develop more NEA detection telescopes.

In Japan, we have set up a non-profit organization, the Japan Spaceguard Association, and lobbied for new NEA telescopes. These activities brought successful results and now new NEA telescopes are under construction at the Bisei Spaceguard Center (Figure 1) (Isobe 2000; Isobe and Williams 2000; Isobe and Japan Spaceguard Association 2000). There are three telescopes with wide fields of view: $25 \mathrm{~cm}$ and $50 \mathrm{~cm}$ telescopes, with a two degree field (Figure 2) and $100 \mathrm{~cm}$ telescope with a three degree field. The first and second telescopes are being tested and have so far detected over one hundred new asteroids. The third telescope will begin operation in summer, 2001.

Table 1 shows some parameters of the $100 \mathrm{~cm}$ telescope which has a size for the focal plane of $162 \mathrm{~mm}$ and 3 degree. To cover this large field, $102 \mathrm{k}$ $\mathrm{x} 4 \mathrm{k}$ CCD chips are set as shown in figure 3. Our target magnitude is 20.5, with an exposure time of about 30 seconds. Considering the read-out time, the data-transfer time, and the telescope pointing time, one should get $160 \mathrm{Mbyte}$ of data per minute, which gives about 2 Tera-byte per month. This number is very big and therefore we are developing automatic reduction software.

We are interested in detecting NEAs in our observational data. However, our data contains much more information which is astronomically useful. In 


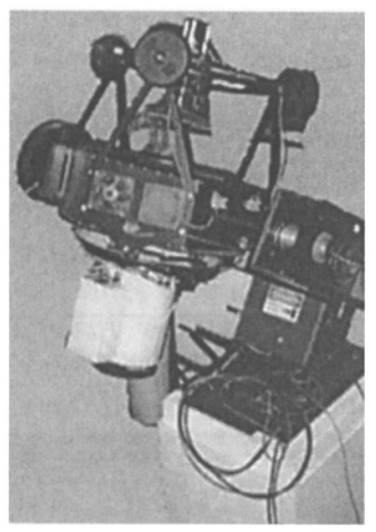

Figure 2. A view of the 50-cm telescope having a short focal length.

Table 1. Some specific parameters of the 1-m telescope.

\begin{tabular}{|l|l|}
\hline Primary mirror & $100 \mathrm{~cm}$ \\
\hline Focal ratio & $\mathrm{F} / 3$ \\
\hline Focal plane size & $162 \mathrm{~mm}$ \\
\hline Field size & $3^{\circ}$ \\
\hline Spectral range & $470 \mathrm{~nm}-800 \mathrm{~nm}$ \\
\hline Point source energy within $15 \mu \mathrm{m} \times 15 \mu \mathrm{m}$ & $80 \%$ \\
\hline Typical image size at the site & 2 arc seconds \\
\hline CCD pixel size & $15 \mu \mathrm{m}$ \\
\hline Focal length & $3 \mathrm{~m}$ \\
\hline Diameter of a 1 arc second image & $15 \mu \mathrm{m}$ \\
\hline CCD pixel number & $2 \mathrm{k} \times 4 \mathrm{k}$ \\
\hline Number of CCD chips covering $3^{\circ}$ field & 10 \\
\hline
\end{tabular}

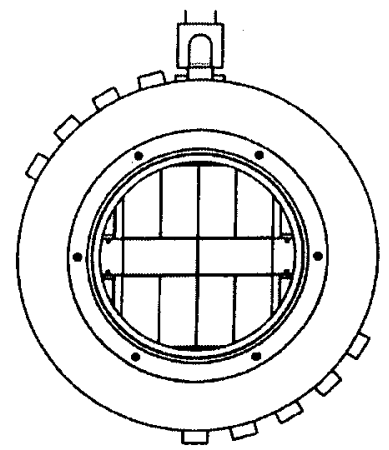

Figure 3. A distribution of $2 \mathrm{k} \times 4 \mathrm{k}$ back-illuminated SITe CCD chips covering 3 degree field. 
Table 2. Schedule of data distribution.

\begin{tabular}{|r|l|}
\hline \multicolumn{2}{|c|}{ Data Distribution } \\
\hline $0-1$ day & Detection of NEAs and Space Debris \\
$1-14$ days & Educational Program \\
$15-28$ days & Open the Data to Public \\
28 days & Delete the Data \\
\hline
\end{tabular}

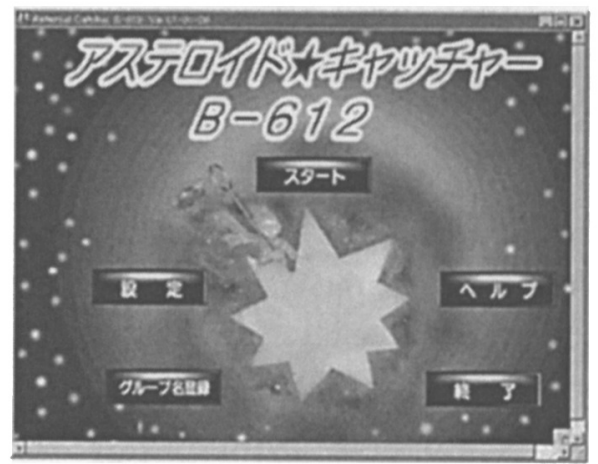

Figure 4. The front page of the software "Asteroid Catcher B-612".

recent years, there has been much development in high speed data transfer systems through Internet and fiber optic lines. Therefore, we decided to distribute the data as outlined in Table 2. We will release all the data two weeks after the observations to registered scientists. During these two weeks, the data will be used for educational programs.

\section{An Educational Program Using the NEA Observational Data}

The idea of our software for educational program is simple. For the NEA observations we usually take 30 second exposures several (3 to 5 ) times with an interval of 15 minutes to 1 hour for a celestial field. One downloads these image data into the memory of a computer and blinks these images after adjusting stellar positions top on top. Moving or variable objects are easily identified within the star field.

To do this properly, one has to develop a system with 1) a fiber optics line, 2) a user's registration home page, 3) a software to identify which team gets which sets of data, 4) a software manual, and 5) a parameter summary of detected objects.

1) The Japan Global Network (JGN) project started a few years ago. The Okayama prefecture where our telescopes are located is expanding its own Okayama Giga-bit Network (OGN) connected to the JGN. Fortunately, this OGN passes close to our site and we can connect our system to it with a small 
amount of cost. It has a capacity of $600 \mathrm{Mbps}$ which is enough for our data transfer.

2) The goal of our homepage is not only to publicize our program but also to identify each applicant and his category of data access right. Certainly, this process needs some minimal manual actions.

3) After a team obtained an identification number and password, they can request our data. However, since there is a criteria for data distribution as shown in Table 2, we have to identify what class of data access right they have.

4) A team can see a data list of exposure images including observing dates, exposure times, filters, central right ascensions and declinations, etc. and then requests a preview image since the whole image would be too large. After choosing the necessary image(s), they can down-load the image data through the Internet. For educational program, we are preparing a software called "Asteroid Catcher B-612" (Figure 4), which is used to detect moving objects and which also contains a manual describing how to use the software.

5) Detected positions of the moving objects should be relayed to the Minor Planet Center (MPC) of the International Astronomical Union (IAU) through the JSGA. All the information should match the format of the MPC well. Our software shows school pupils and the general public how to write up the information and to send it to us.

All this is shown on our homepage : http://www.spaceguard.or.jp.

\section{Methods to Publicize our Program}

The JSGA has its own homepage and a program "Spaceguard Detective Agency Tracking down the asteroids" and tries to publicize it through public lectures and international conferences. To make our program more efficient, we collaborate with the Liverpool John Moore University (JMU) with an interface of the British Council. The JMU is developing a $2 \mathrm{~m}$ robotic telescope in Canarie Island and an educational software. Both teams share their own software and programs. The British Council organized two international meetings, an Asian one in Kuala Lumpur and a European one in Liverpool, in 2000, where representatives from 14 countries got detailed information about the joint project called the International Schools' Observatory (ISO) (Figure 5).

The program will start in the summer of 2001 and some test programs are already underway. In Japan, we will invite 500 groups of school pupils and public people to join the program with collaboration of the Yomiuri Newspaper Co. Ltd, and as a test program, they will get all the software and image data on CD-ROMs.

After this is successful, many people will know about our program and hopefully will decide to join. At the full operation phase, we have the possibility of distributing to nearly 500 teams per full clear night.

\section{Conclusion}

All the teams which join this program can handle real astronomical data and have a high possibility to detect new asteroids. Then, they can get names of 

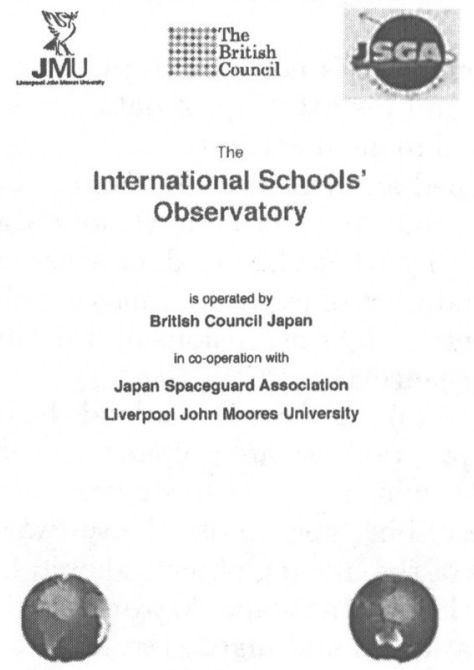

Figure 5. A front page of the program "International Schools' observatory", which is now under development.

discoverers for those objects. It is an exciting experience and can be a real scientific education tool. We are intending to proceed with this program on an international basis.

\section{References}

Isobe, S. 2000, The position of the Japan Spaceguard Association with regard to NEO problems, Planetary and Space Science, 48, 793-795

Isobe, S. and Williams, R. 2000, Education and research opportunities for mining the data of the Bisei Spaceguard Center, in Proceedings of SPIE, Vol. $4010,168-171$

Isobe, S. and Japan Spaceguard Association 2000, Japanese $0.5 \mathrm{~m}$ and $1.0 \mathrm{~m}$ telescopes for near-earth asteroid observations, Ap\&SS, 273, 121-126 